\title{
Uso del bambú como material de construcción en estructuras no convencionales en la ciudad de Huancayo
}

\author{
Bamboo as a building material in unconventional \\ structures in Huancayo city
}

\author{
Vadimir Simón Montoya Torres \\ Universidad Continental \\ vlad00800@yahoo.es
}

\section{RESUMEN}

El objetivo logrado ha sido la experimentación del uso del bambú como material de construcción en estructuras que generen espacios no convencionales. Esta investigación fue de nivel descriptivo, diseño transversal; se aplicaron mediciones de porcentaje de humedad en el bambú con el propósito de medir su resistencia en climas de aire seco. Se escogió la ciudad de Chanchamayo como fuente de materia prima, y para el proceso constructivo se eligió la ciudad de Huancayo, ambas provincias del departamento de Junín. Como resultados de este trabajo, se ha seleccionado material para la construcción, luego se procedió a seccionar las muestras que presentaban un $80 \%$ de humedad en sus fibras, al finalizar el proceso constructivo evidenciaba que la humedad bajó a un 60 \%; luego se construyó una estructura sencilla usando herramientas manuales para ensamblar un paraboloide hiperbólico utilizando la especie de bambú (Guadua angustifolia Kunth). Entre las conclusiones a las cuales se arribó, podemos mencionar que, se logró construir una propuesta geométrica no convencional de manera sencilla y rápida, proponiendo así una estructura no convencional; se desarrolló un rápido ensamble, y su edificación fue favorable al momento de construir; el material sufrió una pérdida de humedad provocada por el clima seco de la ciudad de Huancayo, situación que generó fisuras en las superficies del bambú utilizado, esto demuestra que el material es vulnerable a cambios de espacio geográfico y clima.

\section{ABSTRACT}

The study aim was to experiment with bamboo as a building material to assemble structures that generate unconventional spaces. This paper was of descriptive level, and cross-sectional design; measurements of humidity in bamboo were applied in order to measure their strength in dry air climates. Chanchamayo city was chosen as a source of raw material, and Huancayo city for the construction process, both provinces of Junin region. After selecting the construction material, we sectioned the samples that presented $80 \%$ moisture on its fibers; at the end of the construction process it was evident that moisture lowered down to $60 \%$. As a result; a simple structure made of the bamboo species (Guadua angustifolia Kunth) was built using hand tools to construct a hyperbolic paraboloid. In this way, we managed to build a simple and fast geometric unconventional structure, of quick assembly, and favorable construction when building; the material suffered a loss of moisture caused by the dry climate of the city of Huancayo, thus generating cracks on the bamboo surfaces, and this shows that the material is vulnerable to climate changes and geographical space.

Keywords: Bamboo, guadua, construction, structures, unconventional.

Palabras clave: Bambú, guadua, construcción, estructuras, no convencional.

Historial del artículo:

Recibido: 28 de octubre de 2014. Aprobado: 06 de abril de 2015. Disponible en línea: 30 de junio de 2015

Arquitecto, docente de la Escuela Académico Profesional de Arquitectura de la Universidad Continental 


\section{INTRODUCCIÓN}

Esta investigación propone explorar bajo la viabilidad de una estrategia metodológica de observación y experimentación, las propiedades y virtudes del bambú como una alternativa de sistema constructivo no convencional, obteniendo la materia prima de experimentación de la selva central del Perú, específicamente de la provincia de Chanchamayo, departamento de Junín.

Plantea valorar el conocimiento de nuevas alternativas

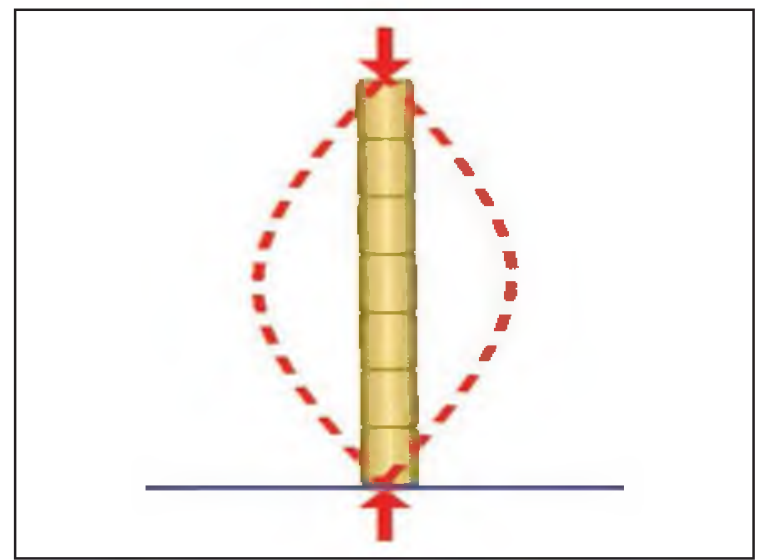

Figura $N^{\circ}$ 1: Esquema de Guadua sometida a compresión.

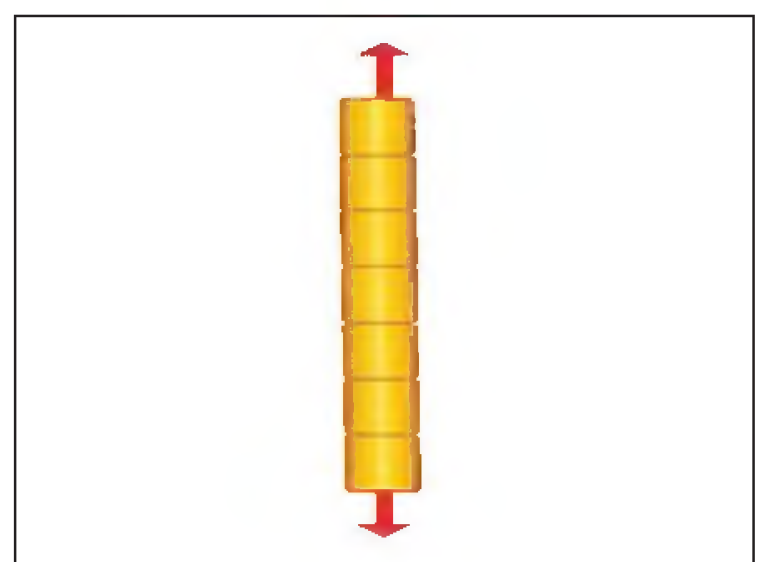

Figura $N^{\circ} 2$ : Esquema de Guadua sometida a tracción.

constructivas, cuya propuesta considera ser sostenibles en su producción, en su aplicación a la construcción, pero que para ello es fundamental primero conocer todas sus virtudes y defectos, y así entender sus posibilidades en el campo constructivo, abriendo un abanico de posibilidades como en lo industrial, artesanal, agrícola, turístico y ecológico.

En la actualidad el Perú pertenece al INBAR (International Network for Bamboo and Rattan), y mediante esta organización mundial se han dado pasos importantes en el desarrollo del bambú en sus diferentes usos. En nuestro caso lo llevamos al campo de la construcción, que es un escenario de múltiples posibilidades, ya el 2008 se dio un gran avance en la legislación peruana, al conocerse la aprobación de Planes Nacionales de Promoción de Caña Brava y Bambú (1) Posteriormente, en el 2012 fue aprobada la Norma Técnica E.0100 del Reglamento Nacional de Construcciones (2), documento de carácter oficial para todo tipo de edificaciones en el país.

El problema planteado fue: ¿̇Cuáles son las limitaciones para construir con bambú estructuras no convencionales en Huancayo? El objetivo fue construir un modelo geométrico a escala real para ensayar los distintos ensambles y empalmes estructurales que se desarrollen dentro del clima de la ciudad de Huancayo y poder realizar mediciones de humedad y de cálculo estructural en función de masa, diámetro y porcentaje de humedad por segmento.

La idea fue desarrollar un modelo construido de una forma geométrica como la de un paraboloide hiperbólico, el cual estará expuesto al medio ambiente, y podremos observar su comportamiento y variación de sus propiedades físico-mecánicas en función del clima.

En lo personal estos ensayos también me permitieron experimentar las posibilidades de transformación de las cañas de bambú en elementos estructurales, que son formulados en gabinete de manera teórica y que por intermedio de esta investigación fueron propuestos en una construcción real y sometida a las condiciones reales con lo que pude entender cómo se comporta el material en función de su disposición estructural y sus posibilidades espaciales.

\section{MATERIAL Y MÉTODOS}

El tipo de investigación propuesto fue descriptivo. El modelo construido a escala real fue realizado por estudiantes de la Escuela Académico Profesional de Arquitectura que nunca habían trabajado con un material como el bambú.

Analizar los rendimientos físicos mecánicos de acuerdo con los estándares de resistencia de la Guadua angustifolia en función de los diámetros de cada caña, es importante hacerlo en rendimientos a los que serán sometidos estos elementos como son los esfuerzos de compresión, flexión, tracción corte e inercia. Estos esfuerzos son analizados de la siguiente manera:

Compresión: El esfuerzo de compresión es resultante de las presiones que existe dentro de un sólido deformable o medio continuo, caracterizada porque tiende a una reducción de volumen o un acortamiento en determinada dirección (figura $\mathrm{N}^{\circ} 1$ ). 
Tracción: En el cálculo de estructuras e ingeniería se denomina tracción al esfuerzo a que está sometido un cuerpo por la aplicación de dos fuerzas que actúan en sentido opuesto, y tienden a estirarlo. Lógicamente, se considera que las tensiones que tiene cualquier sección perpendicular a dichas fuerzas son normales a esa sección, y poseen sentidos opuestos a las fuerzas que intentan alargar el cuerpo (figura $\mathrm{N}^{\circ} 2$ ).

Flexión: Se denomina flexión al tipo de deformación que presenta un elemento estructural alargado en una dirección perpendicular a su eje longitudinal. El término "alargado" se aplica cuando una dimensión es dominante frente a las otras. Un caso típico son las vigas, las que están diseñadas para trabajar, principalmente, por flexión. De igual modo, el concepto de flexión se extiende a elementos estructurales superficiales como placas o láminas.

El rasgo más destacado es que un objeto sometido a

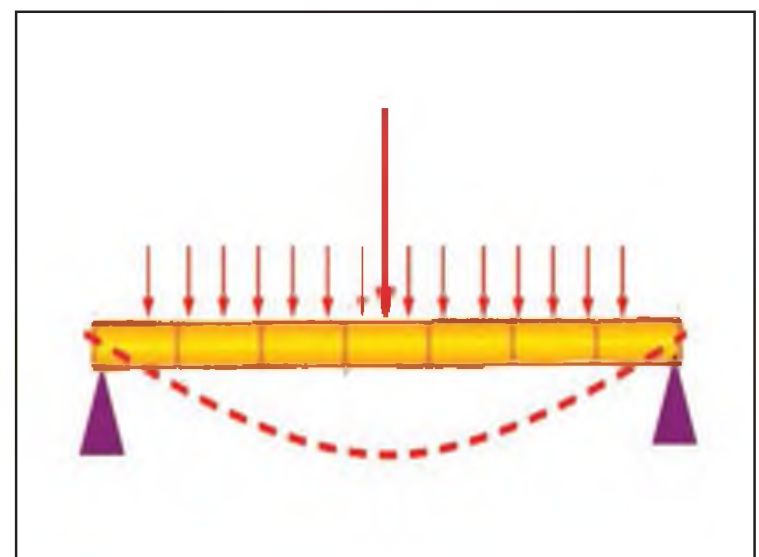

Figura $N^{0} 3$ : Esquema de Guadua sometida a esfuerzos de flexión.

flexión presenta una superficie de puntos tal, llamada fibra neutra, que la distancia a lo largo de cualquier curva contenida en ella no varía con respecto al valor antes de la deformación (figura $\mathrm{N}^{\circ} 3$ ). El esfuerzo que provoca la flexión se denomina momento flector.

Inercia: Es la capacidad de un cuerpo de permanecer en estado de reposo o movimiento. La inercia de la sección a mayor distancia de la masa a su centro de gravedad, mayor será su inercia

Conocer estas propiedades físicas de la guadua (3) - bambú es muy importante porque así podemos comprender los esfuerzos que tienen que soportar estos elementos cuando estén dispuestos en una estructura autoportante. Estos datos los analizaremos y ordenaremos en las siguientes tablas, la primera es para calcular el área de la sección de cada bambú (tabla $N^{\circ} 1$ ), con el resultado de este primer cálculo se desarrolla el análisis de capacidades portantes (tabla
$N^{\circ} 2$ ), en el cual se analiza las diferentes muestras de bambú de acuerdo con un proceso metodológico de toma de datos que recomienda los formatos de experimentación con guadua (4).

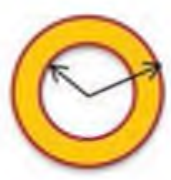

\section{Relación diámetro-resistencia}

Área $=\underline{\pi}\left(R^{2}-r^{2}\right)$

\section{Área de corona circular}

Tabla № 1: Cálculo de área de sección circular de diferentes diámetros de bambúes.

\begin{tabular}{ccccc}
\hline Guadua & R en cm. & r en cm. & Espesor & $\begin{array}{c}\text { Área } \\
\text { en } \mathbf{c m}^{2}\end{array}$ \\
\hline 1 & 4,10 & 3,60 & 1,00 & 12,10 \\
2 & 4,25 & 3,80 & 0,90 & 11,38 \\
3 & 4,31 & 3,85 & 0,92 & 11,79 \\
4 & 4,82 & 3,98 & 1,68 & 23,22 \\
5 & 5,12 & 4,25 & 1,74 & 25,61 \\
6 & 5,42 & 4,61 & 1,62 & 25,52 \\
7 & 5,80 & 4,98 & 1,64 & 27,77 \\
8 & 6,20 & 5,36 & 1,68 & 30,51 \\
9 & 6,40 & 5,58 & 1,64 & 30,86 \\
10 & 6,78 & 5,91 & 1,74 & 34,68 \\
11 & 6,98 & 6,21 & 1,54 & 31,91 \\
12 & 7,28 & 6,49 & 1,58 & 34,18 \\
\hline
\end{tabular}

Tabla N 2: Cálculo de resistencias de cañas para una carga puntual de acuerdo con la carga admisible.

\begin{tabular}{cccccc}
\hline Guadua & $\begin{array}{c}\text { Ag en } \\
\text { cm }^{2} .\end{array}$ & $\begin{array}{c}\text { Pu en } \\
\text { kg. }\end{array}$ & $\begin{array}{c}\text { Fc en } \\
\mathrm{kg} / \mathrm{cm}^{2}\end{array}$ & Estimado & $\begin{array}{c}\mathbf{n}^{\circ} \text { de } \\
\text { guaduas }\end{array}$ \\
\hline 1 & 12,10 & 5000 & 130 & 3,179 & 3 \\
2 & 11,38 & 5000 & 130 & 3,380 & 3 \\
3 & 11,79 & 5000 & 130 & 3,262 & 3 \\
4 & 23,22 & 5000 & 130 & 1,656 & 2 \\
5 & 25,61 & 5000 & 130 & 1,502 & 2 \\
6 & 25,52 & 5000 & 130 & 1,507 & 2 \\
7 & 27,77 & 5000 & 130 & 1,385 & 1 \\
8 & 30,51 & 5000 & 130 & 1,261 & 1 \\
9 & 30,86 & 5000 & 130 & 1,246 & 1 \\
10 & 34,68 & 5000 & 130 & 1,109 & 1 \\
11 & 31,91 & 5000 & 130 & 1,205 & 1 \\
12 & 34,18 & 5000 & 130 & 1,125 & 1 \\
\hline
\end{tabular}

Para el segundo cálculo usamos la siguiente lógica, siendo el caso de soportar una carga tributaria de 5 $000 \mathrm{~kg}$ aplicamos la fórmula de elementos sometidos a compresión para determinar el número de guaduas para una columna. 


$$
N_{c}=\frac{P u}{A g \times F_{c}}
$$

Dónde:

$N_{c}=$ Numero de guaduas en la columna

$\mathrm{Pu}=$ Carga tributaria de la columna

$\mathrm{Ag}=$ Área de las guaduas.

$\mathrm{Fc}_{\mathrm{c}}=$ Esfuerzo admisible a esfuerzos de compresión (130 kg/cm2)

En su hábitat natural los bambúes pueden crecer de rizomas o raíces, que es muy importante, porque esta planta no tiene un tallo central como en el caso de los árboles que cuentan con una sola raíz, esta diferencia hace que los bambúes se sustenten en sus rizomas, como un sistema subterráneo de soporte, esto permite un crecimiento acelerado de la especie, que una para proteger este producto natural de los efectos de la humedad y proteger del ataque de los insectos y la descomposición por hongos. El bambú no tiene ninguna resina o ácido tánico, por lo que es muy adecuado para pintar (6).

Algunas especies de bambúes son las plantas terrestres conocidas de crecimiento más veloz ya que se observan bambúes que crecen $1 \mathrm{~m}$ en $24 \mathrm{~h}$, pero en condiciones de laboratorio. Algunos géneros importantes de bambúes leñosos son Bambusa (120 especies), Chusquea (100 especies) y Phyllostachys (45 especies) (7).

\section{RESULTADOS}

Se logró construir un paraboloide hiperbólico de $4 \mathrm{~m}$

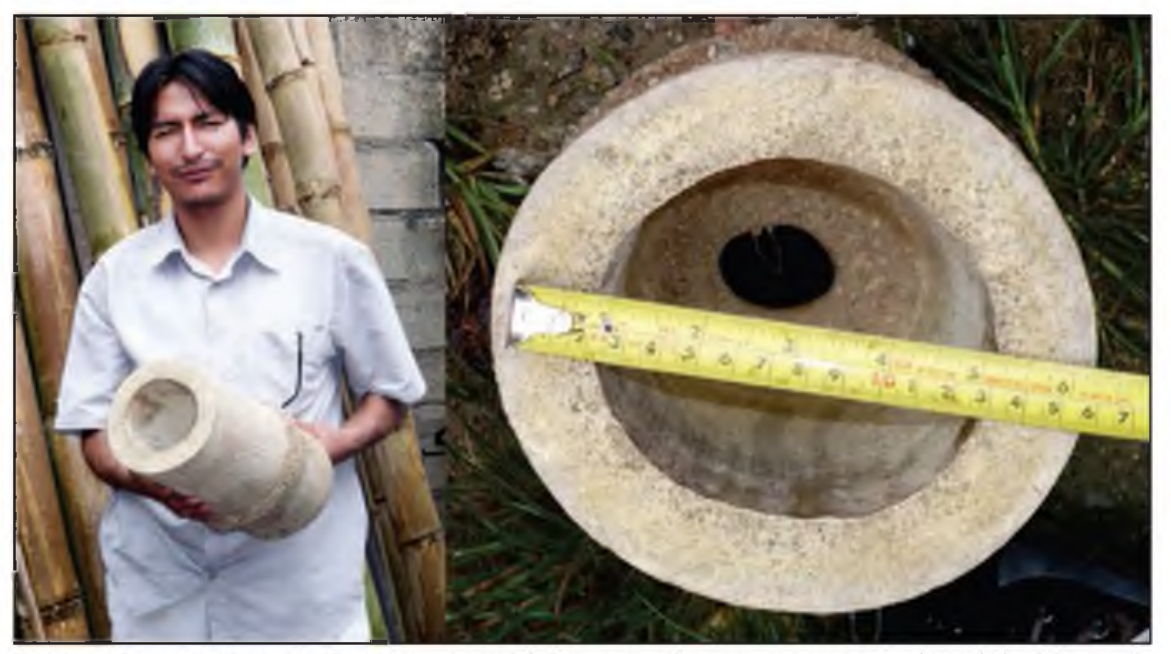

Figura $N^{\circ} 4$ : Muestra de Guadua angustifolia, que alcanza una sección basal de $16 \mathrm{~cm}$ de diámetro.

vez conformado sus sistema de rizomas, crece de manera aleatoria dentro de la red subterránea, lo cual evidencia su crecimiento tipo gramínea (5).

Los bambúes en muchas partes del mundo pueden crecer hasta una altura cercana de $25 \mathrm{~m}$ con un diámetro considerablemente muy estrecho, su madera es $25 \%$ más dura que el roble y es más durable que muchas de las especies de maderas duras y de clasificación A. El interior hueco hace que sea un material altamente ligero y elástico, en promedio de $600-800 \mathrm{~kg} / \mathrm{m} 3$.

La fuerza de flexión extrema y una resistencia a la tracción parecida a las estructuras de acero hace del bambú una elección obvia como un material de construcción resistente a las fuerzas sísmicas, sin embargo puede astillarse cuando se utiliza en espacios exteriores expuestos, también es necesario de lado, el cual fue ensamblado con la participación de estudiantes de la Escuela Académico Profesional de Arquitectura de la Universidad Continental y dentro del campus universitario (figura $\mathrm{N}^{\circ} 5$ y 6 ).

Esta estructura trabajó en un proceso de tres meses, iniciado con el traslado de las cañas de bambú desde la ciudad de La Merced, Chanchamayo, hacia la ciudad de Huancayo en un estado verde y con un alto porcentaje de humedad, el cual se fue disipando rápidamente por el cambio de clima (figura $\mathrm{N}^{\circ}$ 7).

Las estructuras se ensamblaron con facilidad por la ventaja del material de ser liviano sobre el volumen que ocupa. Esta característica se acentuó más con los días de trabajo a razón de la deshidratación de las cañas de bambú. Durante el trabajo se usaron herramientas manuales con las que se transformó los culmos de bambú en latillas longitudinales, las cuales adoptan 


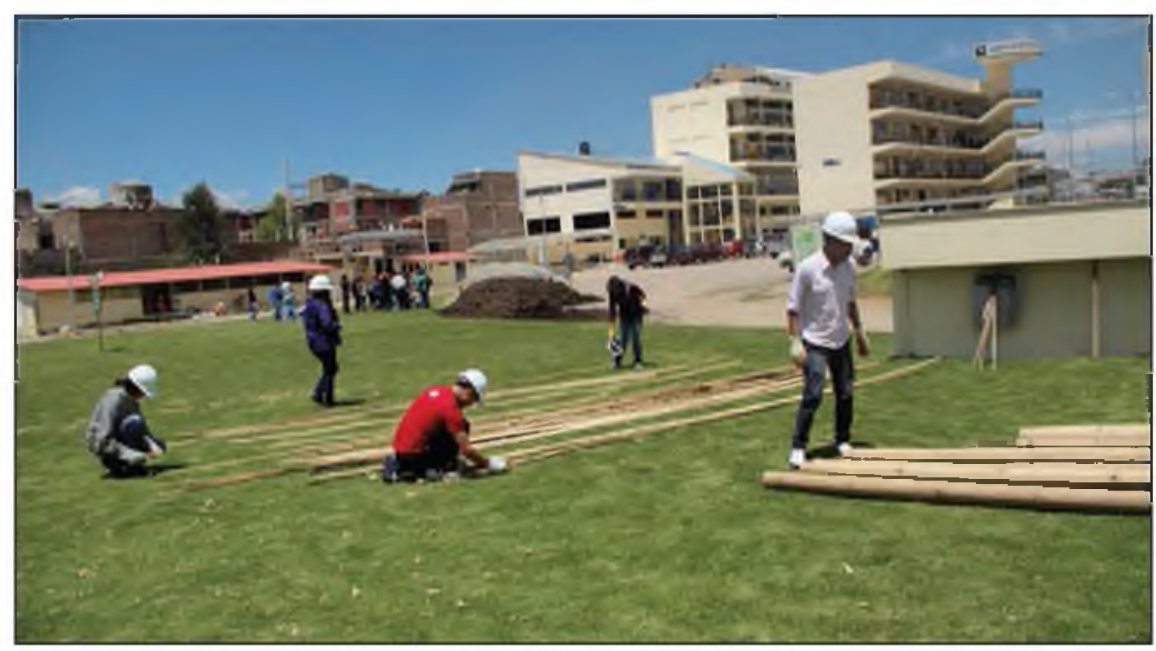

Figura $N^{\circ} 5 \mathrm{~A}$ : Construcción de la estructura del paraboloide hiperbólico.

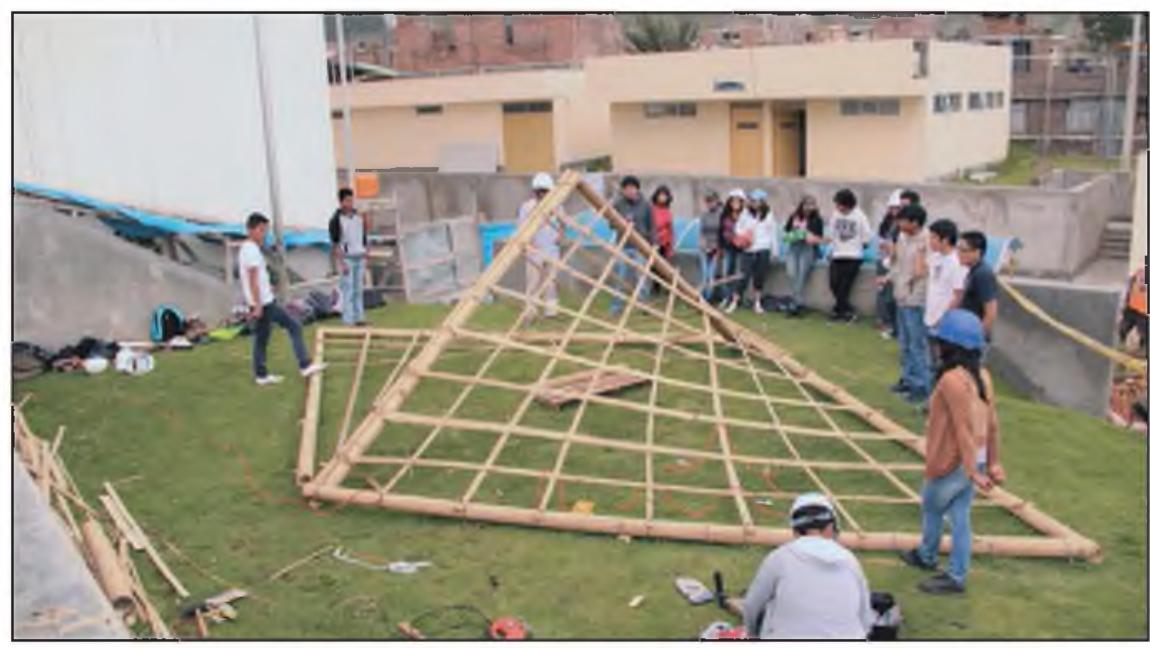

Figura $N^{\circ} 5 B$ : Construcción de la estructura del paraboloide hiperbólico.

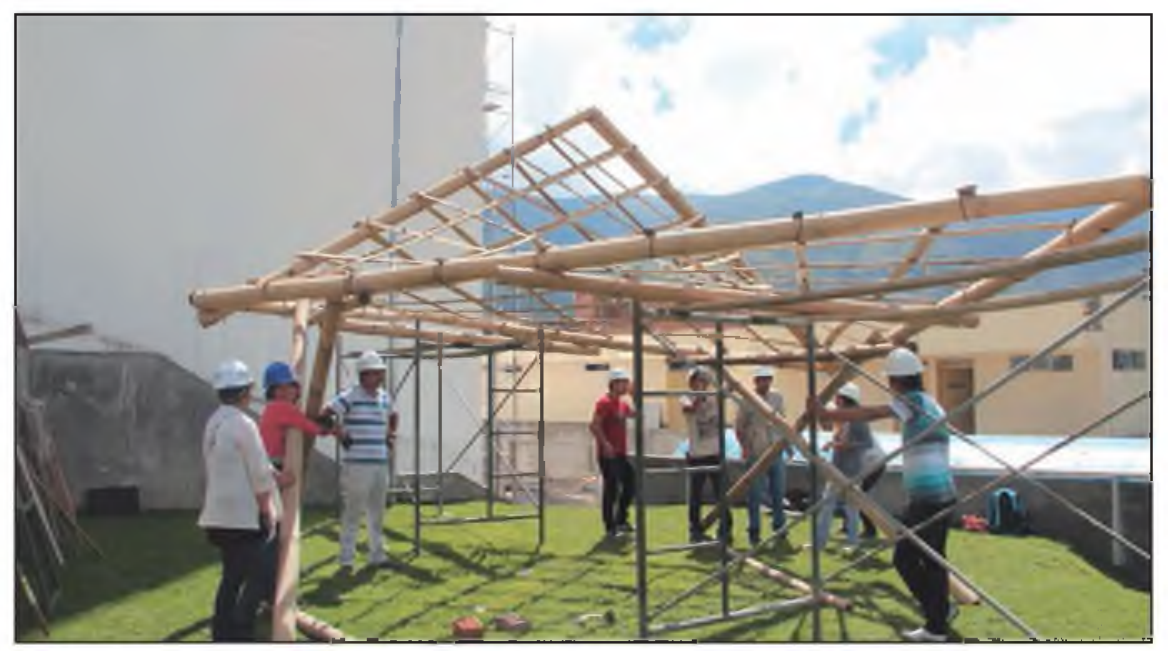

Figura $N^{\circ} 5 \mathrm{C}$ : Construcción de la estructura del paraboloide hiperbólico. 


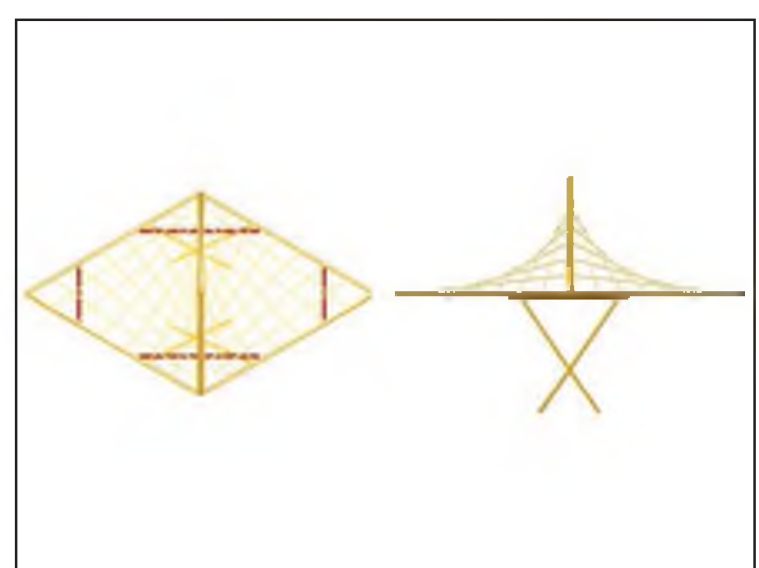

Figura $\mathrm{N}^{\circ}$ 6: Vistas de la estructura del paraboloide hiperbólico.

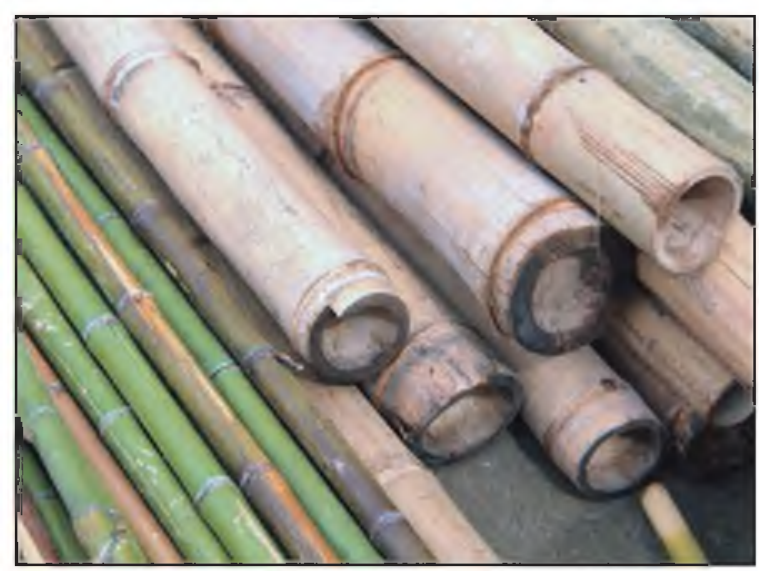

Figura $N^{0} 7$ : Cañas de bambú en su estado postraslado.

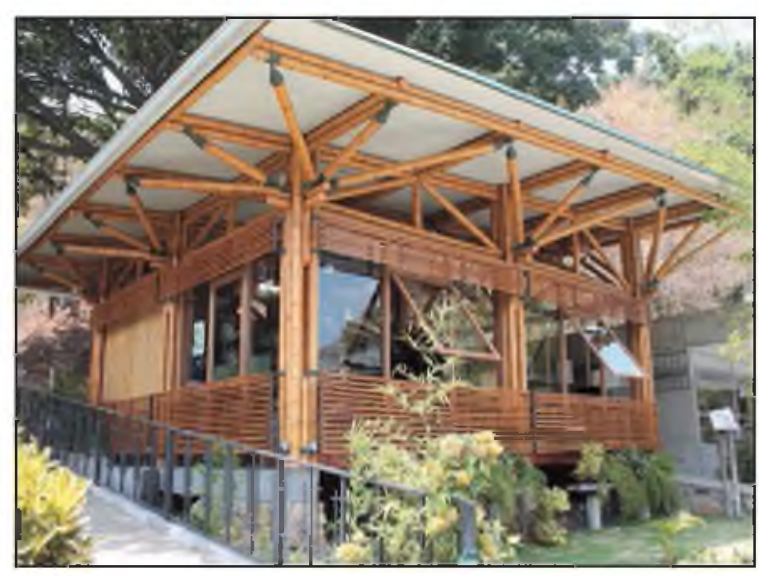

Figura $\mathrm{N}^{\circ}$ 8: Biblioteca y Centro de Investigación de Bambú de la Universidad Santiago Católica de Guayaquil, Ecuador.

una gran flexibilidad con respecto a su configuración tubular. Posterior al trabajo de habilitado de culmos y de latillas se utilizaron herramientas eléctricas para generar perforaciones y usar pernos y varillas roscadas para empalmar los componentes en la estructura. Este método de ensamble es recogido de las experiencias colombianas y ecuatorianas (3) de técnicas de uniones ancestrales (8) y bajo las recomendaciones en el desarrollo de uniones tecnificadas por las experiencias ecuatorianas de ensamble de estructuras de bambú.

\section{DISCUSIÓN}

Los resultados obtenidos de esta experimentación de técnicas constructivas con bambú fueron favorables en el aspecto de versatilidad y facilidad para desarrollar los ensambles, ello se pudo evidenciar al trabajar con los estudiantes, quienes comprendieron y aprendieron rápidamente cómo manejar el sistema constructivo.

Otro factor importante es el comprobar la relación peso versus volumen lo cual justifica lo liviano que es material a pesar de tener un gran volumen (600-800 $\mathrm{kg} / \mathrm{m} 3$ ), esta relación aumenta su valor.

La humedad interna del bambú fue variando con el tiempo, el monitoreo se realizó durante el proceso de la experimentación, inicialmente este es un factor muy favorable pero al pasar los días el factor de humedad en el interior de las fibras del bambú se acrecienta y esta pérdida de humedad causada por el clima seco de la ciudad de Huancayo se manifiesta negativamente al generar fisuras en las superficies de los culmos (entrenudos) del bambú, que primero son fisuras leves, pero al pasar las semanas estas fisuras se extienden por el resto de la caña. Este fenómeno es causado por la deshidratación excesiva del bambú y se acentúa con mayor fuerza cuando está expuesto a lluvia y sol continuo.

Los bambúes que se encuentran en sombra sufren fisuras pero no en exceso, esto sin considerar que la materia prima no fue preservada como lo recomiendan los estudios de preservación técnica y ancestral del Arq. Jorge Moran Ubidia (9). Este caso fue muy importante observar durante la construcción y tiempo de recolección de datos porque de esta manera comprendemos mejor que un material como el bambú es muy versátil pero no es óptimo en un clima seco como el de la ciudad de Huancayo, donde los cambios de temperatura son muy bruscos y las estaciones climáticas bien marcadas. La experiencia nos encamina a una lógica que los materiales de construcción de una determinada latitud geográfica como es la selva central, se comportan mejor en su clima de origen, para nuestro caso la materia prima fue obtenida de la ciudad de La Merced, Chanchamayo, departamento de Junín, y se trabajó con la especie de Guadua angustifolia Kunth, que de acuerdo con los empadronamientos realizados por INBAR en Latinoamérica, la selva alta del Perú en el departamento de Junín es hábitat natural de esta especie de gramínea. 
El trabajo permitió experimentar de primera mano un material no convencional en un escenario geográfico distinto al de su hábitat, construyendo una estructura autoportante y que se pueda observar sus cambios físicos durante la experimentación.

En conclusión, el bambú es un material que demuestra sus propiedades físicas mecánicas y su amplia versatilidad en la aplicación constructiva de elementos estructurales y que deja muchos más campos de investigación en torno a sus propiedades y a su vulnerabilidades como material natural y orgánico. Es una realidad muy distinta la situación del bambú en nuestro vecino país del norte, el caso de Ecuador que dedica mucho tiempo y esfuerzos económicos a la investigación en bambú (figura $N^{\circ} 8$ ), les ha permitido avanzar y proponer nuevas y propias tecnología. Nuestro caso es que contamos con una gama mayor de variedades y climas donde poder experimentar las diferentes posibilidades del bambú, es un reto que como peruanos debemos alcanzar más aún que ahora ya existe un marco normativo y legislativo.

\section{REFERENCIAS BIBLIOGRÁFICAS}

1. Resolución Ministerial $N^{\circ}$ 0521-2008-AG. Ministerio de Agricultura. Lima, 1 de julio de 2008.

2. Reglamento Nacional de Edificaciones, Norma Técnica E.0100. Ministerio de Vivienda, Construcción y Saneamiento. Lima; 2012.

3. Rubio G. Arte y mañas de la guadua. Una guía sobre el uso productivo de un bambú gigante. Bogotá: Info Art; 2007.

4. López L, Silva M. Comportamiento sismorresistente de estructuras en bahereque. Manizales: UNC; 2000.

5. Jenssen J. Designing and building with bamboo. Technical University of Eindhoven. Eindhoven, The Netherlands: INBAR; 2000.

6. Peters S. Material revolution susteaintable and multi purpose materials for design And architecture. Alemania: Birkhäuser; 2013

7. Yang Y. Bambúes exóticos: guía para facilitar su identificación. Corporación Botánica. Ecuador: INBAR-LAC; 2006.

8. Hidalgo O. Bamboo, the gift of the gods. Bogotá: D'VINNI; 2003.

9. Moran J. Building with bamboo doing your self manual. Ecuador: INBAR-LAC; 2001. 